\title{
The effect of apocynin on motor and cognitive functions in experimental Alzheimer's disease.
}

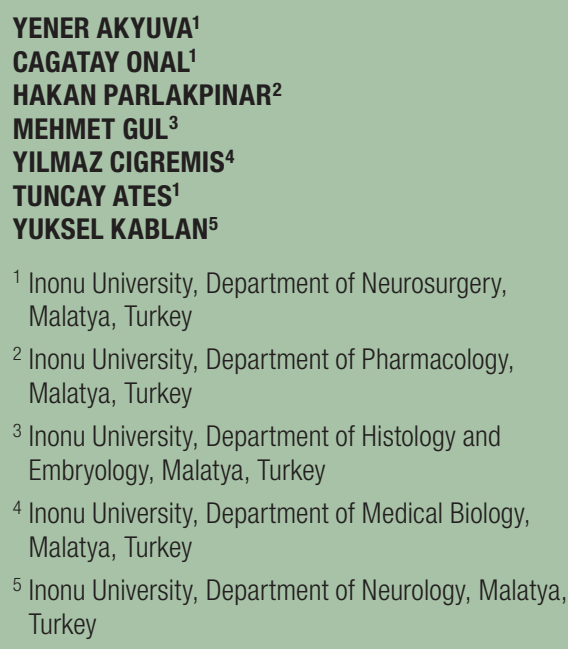

\begin{abstract}
Scope: We investigated the potential beneficial effect of Apocynin (APO) on motor and cognitive functions in experimental Alzheimer's disease (AD).

Materials and Methods: Experimental $A D$ was induced in rats by intraventricular streptozotocin (STZ) injection. Sham group received artificial cerebrospinal fluid (CSF). Both groups were randomly divided into two subgroups. One of the subgroups received intraperitoneal $A P O$ for while the other had normal saline (NS). The animals were evaluated with rotarod, accelerod and Water-Maze tests before and after the treatment. Additionally, biochemical markers of oxidative stress such as malondialdehyde (MDA) and reduced glutathione (GSH) were analyzed from brain specimens. Standard histological evaluation and transmission electron microscopy (TEM) were used to evaluate the neural damage.
\end{abstract}

Results: The difference between STZ+NS in comparison with CSF+NS, $C S F+A P O$ and $S T Z+A P O$ were statistically significant on 30 and $40 \mathrm{rpm}$ on rotarod test. GSH levels, accelerod and Water-Maze test results were not statistically significant between subgroups. However, MDA differences between $S T Z+N S$ in comparison with $C S F+N S, C S F+A P O$ and $S T Z+A P O$ were statistically significant. Hemotoxilene eozine staining and TEM results showed apocynins protective effect.

Conclusion: These results indicate that APO can provide neuro-protective effect for motor but not for cognitive performance in experimental $A D$.

\section{INTRODUCTION}

Inflammation has been shown to play a vital role in the pathophysiology of the Alzheimer's disease (AD). Microglial cells are responsible for removing the remnants of apoptotic neurons and by doing so they induce an inflammatory response against amyloid plaques via oxidative stress. This theory claims that oxidative stress is the main damaging factor in $\operatorname{AD}(1,2,3)$. However, antioxidant molecules have not shown to demonstrate significant clinical benefits $(2,4)$.

Recent researches indicate that $\mathrm{AD}$ is a brain-specific form of diabetes $(5,6)$. Hyperglycemia and hyperinsulinemia are correlated with development of AD-related pathology $(6,7)$. Defective insulin signaling, altered levels and/or abnormal activation of components of the insulin signaling pathway, and, more importantly, decreased responsiveness to insulin were found in $\mathrm{AD}(5,8)$. In response to infection, trauma or abnormal collection of protein aggregates; TNF- $\alpha$ is produced mainly from microglial cells in the brain. TNF- $\alpha$ levels are increased in AD 
brain and cerebrospinal fluid. Impaired neuronal insulin signaling in $\mathrm{AD}$ is connected to that soluble proteins of the amyloid- $\beta(\mathrm{A} \beta)$ peptide-synaptotoxins accumulate in $\mathrm{AD}$ and cause insulin receptor substrate-1 inhibition through TNF- $\alpha$ activation $(9,10)$.

Intraventricular STZ effectively blocks insulin receptors, deteriorates the glucose metabolism and leads to progressive cognitive impairment. STZ induces free radical and hydrogen peroxide $\left(\mathrm{H}_{2} \mathrm{O}_{2}\right)$ generation, ATP depletion, DNA damage in insulin secreting cells as well as decrement of insulin receptor auto-phosphorylation followed by insulin resistance in insulin receptor expressing cells (11). In the brain the main mechanism of action is the development of oxidative stress, mitochondrial dysfunction and activation of the apoptotic pathway (12). Intraventricular STZ injection is one of the most frequently used models of experimental AD (13).

Apocynin (APO) is a naturally occurring NAPDH oxidase inhibitor obtained from the roots of Apocynum cannabinum (14). APO was shown to implement its antiinflammatory function through the blockage of free radical, oxygen ion and peroxide formation. The biochemical basis is the inhibition of NADPH oxidase that reduces oxygen to superoxide radical $(14,15)$. It has demonstrated beneficial effects in different experimental inflammatory, neurodegenerative and ischemic brain diseases $(16,17)$. In this study, we aimed to research the effect of APO on STZ-induced experimental AD.

\section{MATERIALS AND METHODS}

\section{Experimental animals}

The Ethics Committee approved this study (Date:25/11/2013, Protocol number:2013/A-86). Seventy (35 male, 35 female) Wistar-Albino rats aged 3-4 months, weighting 200-275 grams were used as experimental animals (50 experimental and 20 sham). Animals were kept under standard conditions according to the guidelines of the National Institutes of Health and Helsinki Declaration.

Before recruiting to the study all potential animals were tested by using rotarod $(5 \mathrm{rpm} 300$ seconds) test. The failed animals were replaced by new rats, which passed the test.

\section{Rotarod and accelerod tests}

We used the previously published standard protocol for the testing $(18,19)$. Rotamex system $4 / 8$ (Columbus Instruments, Columbus, Ohio) was used to assess animals' performance. Rotarod test was performed at 5, 10, 20, 30, and 40 rpm's for maximum 300 seconds. Accelerod test was performed by increasing the speed from 1 to 79 for 240 and 600 seconds (Picture 1A).

\section{Water-Maze test}

The detailed description of the test has been published elsewhere (20). Briefly, the rats were allowed to reach the platform for 90 seconds. If an animal could not find the platform after 90 seconds it was placed on the platform. Then the animals were allowed to stay on the platform for 15 seconds to memorize its location. This procedure was repeated for four consecutive days. On the fifth day, the animals were placed into the pool with underwater platform. A digital camera hanged at the top of the pool recorded the animals' movements. The camera was connected to a computer to record and track animals' movements. The time spent to reach the hidden platform, time on the platform, and the distance covered in 90 seconds were recorded (Figure 1B).

\section{Induction of AD (intraventricular injection)}

The rats were anesthetized by intraperitoneal $10 \mathrm{mg} / \mathrm{kg}$ xylazine and $75 \mathrm{mg} / \mathrm{kg}$ ketamine injections. If needed additional anesthetics were given but not exceeding more than $20 \%$ of the initial dose.

The technique of ventricular cannulation has been reported elsewhere (21). Briefly, the heads were stabilized on the stereotaxic frame (ASI instruments, Istanbul) $5 \mathrm{~mm}$ above the incisor bar interaural line. Under aseptic condition a sagittal incision was made. The bregma and sagittal sutures were exposed. The trajectory was defined by using Cartesian co-ordinate system according to the PaxinosWatson stereotaxic atlas (22). Two mini burr holes were made on either side. The target point was identified 1.5 lateral to the midline $0.8 \mathrm{~mm}$ posterior to the bregma and the depth was $3.5 \mathrm{~mm}$. The experimental group received totally $3 \mathrm{mg} / \mathrm{kg}$ STZ and the control group received $20 \mu \mathrm{l}$ of artificial cerebrospinal fluid (CSF) (Sigma) via Hamiltonian syringe (Picture 1C). The time required to induce neurodegenerative changes was set for 21 days $(21,23)$.

\section{Study plan}

34 rats (68\% from total 50 experimental) and $16(80 \%$ from total 20 sham) groups survived the procedure. These animals were evaluated by Water-Maze, rotarod and accelerod tests. Two rats from the sham group had procedure related hemiparesis and therefore were excluded from the study leaving 14 animals ( 7 male, 7 female) in the sham group ( $70 \%$ from total 20 sham group). In order to test the APO efficacy in the worst-case scenario, we selected 15 animals (6 male, 9 female) from the experimental group with worst performance results to match the sham group. Both groups were randomly divided into two subgroups, which eventually received either intraperitoneal APO or normal saline.

$\mathrm{CSF}+\mathrm{APO}$ and $\mathrm{STZ}+\mathrm{APO}$ subgroups received $10 \mathrm{mg} /$ $\mathrm{kg}$ intraperitoneally APO for 10 consecutive days (approximately $2-2.5 \mathrm{cc}$ ). Control subgroups $-\mathrm{CSF}+\mathrm{NS}$ and 


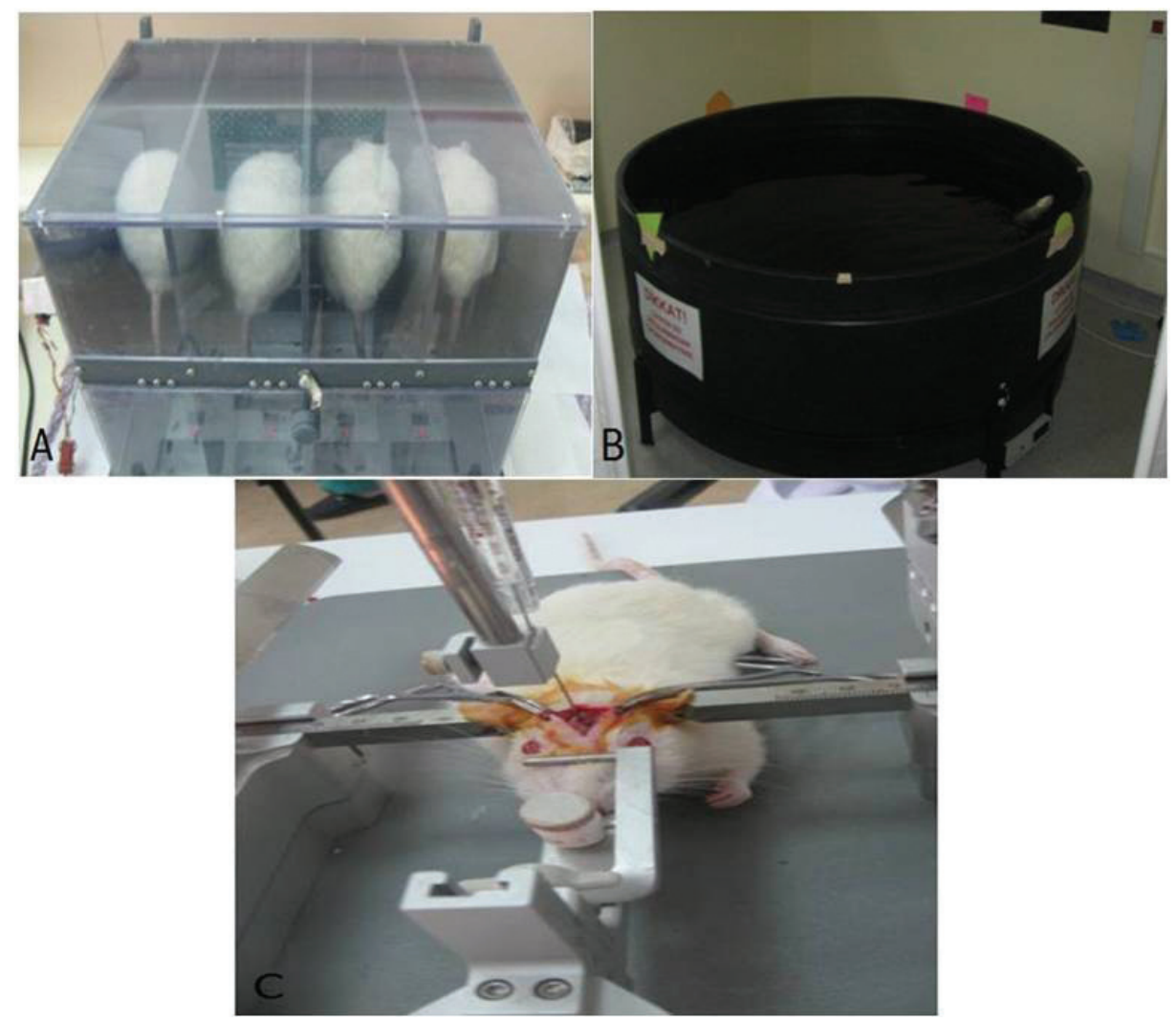

Figure 1: Picture of rotarod test (A), Water-Maze test (B), ICV injection (C)

STZ+NS received the same amount of normal saline intraperitoneally. After 10 treatment days, the animals were tested by Water-Maze, rotarod and accelerod tests.

\section{Biochemical and histological examination}

During sacrification the animals were deeply anesthetized by xylazine and ketamine. Thoracotomy was performed, and blood samples were drawn via intracardial route. These blood samples were used for routine biochemical analysis, which included glucose, blood urea nitrogen, creatinine, aspartate aminotransferase, alanine aminotransferase, sodium, and calcium. The brains were carefully dissected. One hemisphere was used for histological examination and the other was kept at $-70^{\circ} \mathrm{C}$ for biochemical analysis. During biochemical evaluation, the brain samples were weighted, $10 \%$ phosphate tampon $(\mathrm{pH} 7.4,50 \mathrm{mM})$ was added and then homogenated on ice bath for 1-2 minutes at $12000 \mathrm{rpm}$ (Ultra Turrax Type T25-B homogenizer, IKA Labortechnic, Germany). A portion of the homogenate was used to measure MDA level. The rest was centrifuged at $5000 \mathrm{rpm}$ for 30 minutes at $4{ }^{\circ} \mathrm{C}$. The supernatant was drawn and used to measure reduced glutathione (GSH) level.

The brain tissue samples were fixed in 10\% formalin for 48 hours for histopathological examination. After fixation and routine histologic tissue preparation procedures, the cerebral tissue specimens were embedded in paraffin blocks. Sections of $6-8 \mu \mathrm{m}$ thicknesses were prepared using a microtome and stained with hematoxylin and eosin (H-E). Brain sections were examined by a Leica DFC 280 light microscope and Leica Q Win Plus V3 Image Analysis system (Leica Micros Imaging Solutions, Cambridge, UK) and photographed.

The second part $(2 \times 2 \times 2 \mathrm{~mm})$ of the brain tissues was processed for transmission electron microscopic examination (TEM). For that purpose, brain samples were fixed in $2.5 \%$ glutaraldehyde buffered with $0.2 \mathrm{M} \mathrm{NaH} 2 \mathrm{PO} 4$ $+\mathrm{NaHPO} 4(\mathrm{pH} 7.2-7.3)$ and post fixed in $1 \%$ OsO4. After dehydration whit acetone, brain tissue samples were embedded in Araldite CY 212. The ultrathin sections (80 $\mathrm{nm}$ ) taken from the araldite blocks using an ultramicrotome mounted on copper grids. Ultrathin sections were contrasted with uranyl acetate and lead citrate then examined whit Zeiss Libra 120 TEM (Carl Zeiss Company, Oberkochen, Germany) and photographed.

\section{Statistical analysis}

For detecting even minor effects, the required sample sizes used in this study were identified by using statistical power analysis. The sample sizes necessary for a power of 0.80 were estimated using NCSS software. All data were analyzed with a commercially available statistics software 


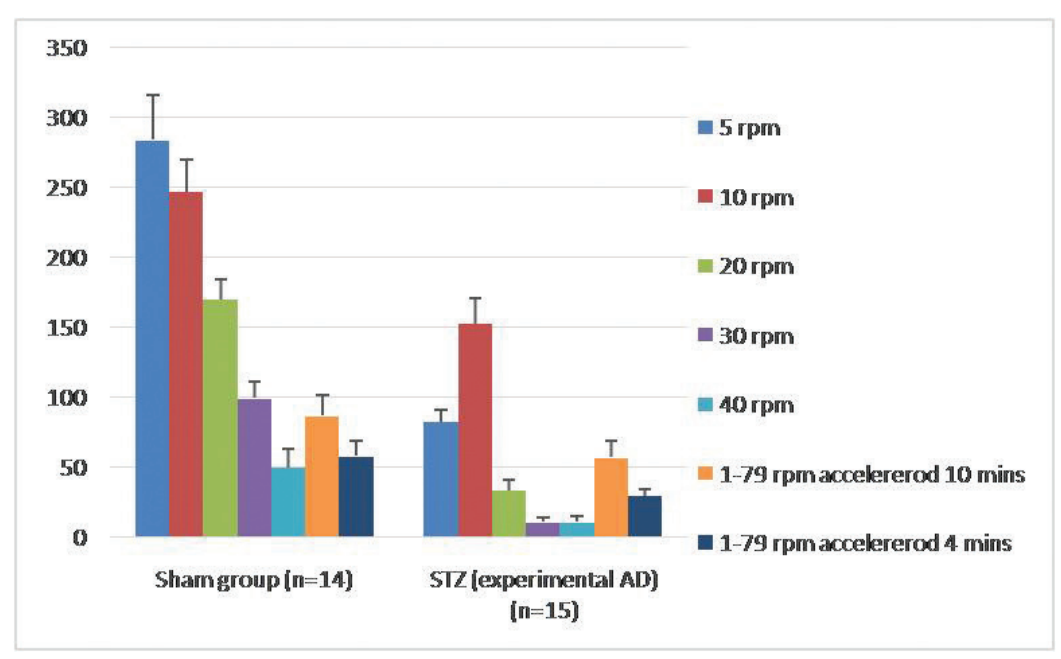

Figure 2. The results of the $S T Z$ and sham groups on rotarod and acclererod tests (5 rpm: $300 \pm$ ) Striking differences in performance is due to detrimental effect of STZ on cognitive functions (vertical axis shown in seconds). The results of the control group were statistically significant compared to the STZ group.

package (SPSS for Windows v.22.0, Chicago, Ilinois, USA). Distributions of the groups were analyzed with the Kolmogorov-Smirnov test. The Kruskal-Wallis H test was performed for comparison of the groups. After the Kruskal-Wallis $\mathrm{H}$ test, multivariate comparisons were carried out by using Student-Newman-Keules test. The data were given as median (min-max). A "P" value of $<0.05$ was considered statistically significant.

\section{RESULTS}

\section{Results rotarod and accelerod tests}

On initial evaluation, there were striking differences between the experimental and sham groups. The differences reached statistical significance on all tests except for accelerod 10 min test (Figure 2). We attributed these results to STZ's detrimental effect on motor and cognitive skills.

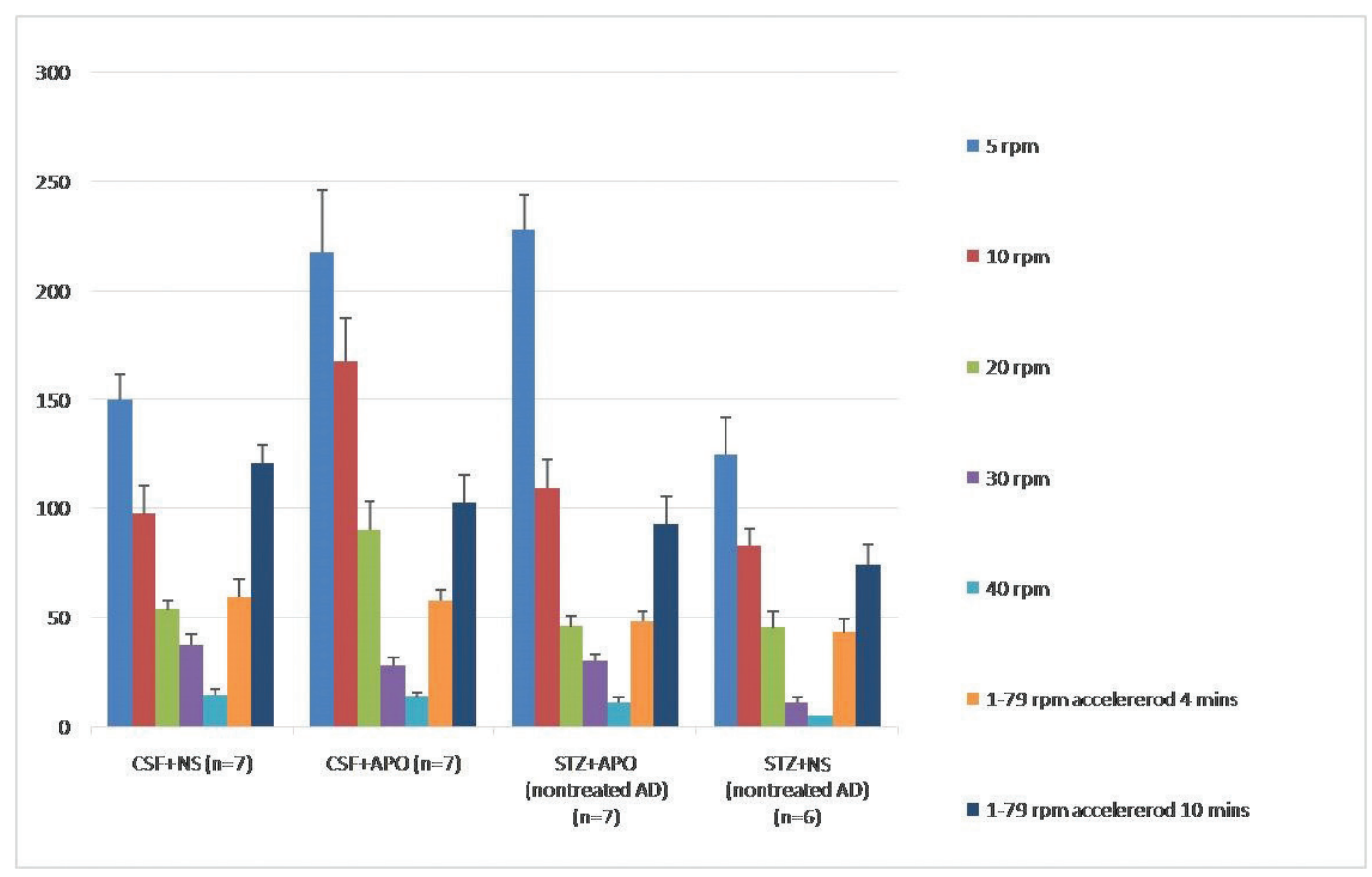

Figure 3. The results of rotarod and accelerod tests. The difference between $S T Z+N S$ in comparison with $C S F+N S, C S F+A P O$ and $S T Z+A P O$ were statistically significant on 30 and $40 \mathrm{rpm}(p<0.05)$. All other comparisons did not reach statistical significance (vertical axis shown in seconds). Higher rotation speed (30 and $40 \mathrm{rpm}$ ) revealed significant differences between $S T Z+N S$ and $C S F+N S, C S F+A P O, S T Z+A P O$ 
On subgroup analysis, however, the difference between $\mathrm{STZ}+\mathrm{NS}$ in comparison with $\mathrm{CSF}+\mathrm{NS}, \mathrm{CSF}+\mathrm{APO}$ and STZ+APO were statistically significant on 30 and $40 \mathrm{rpm}$ $(\mathrm{p}<0.05)$. All other comparisons did not reach statistical significance (Figure 3).

\section{Water-Maze test results}

The differences between the experimental and sham groups reached the statistical significance on time to arrival to the platform $(\mathrm{p}<0.001$, Figure 4$)$.
The subgroup analysis showed no statistically significant differences between any subgroups (Figure 5).

\section{Brain MDA and GSH results}

Blood biochemistry results were within normal limits and the differences were statistically non-significant across the treatment groups.

The differences between the brain's GSH across the treatment groups were not statistically significant. MDA

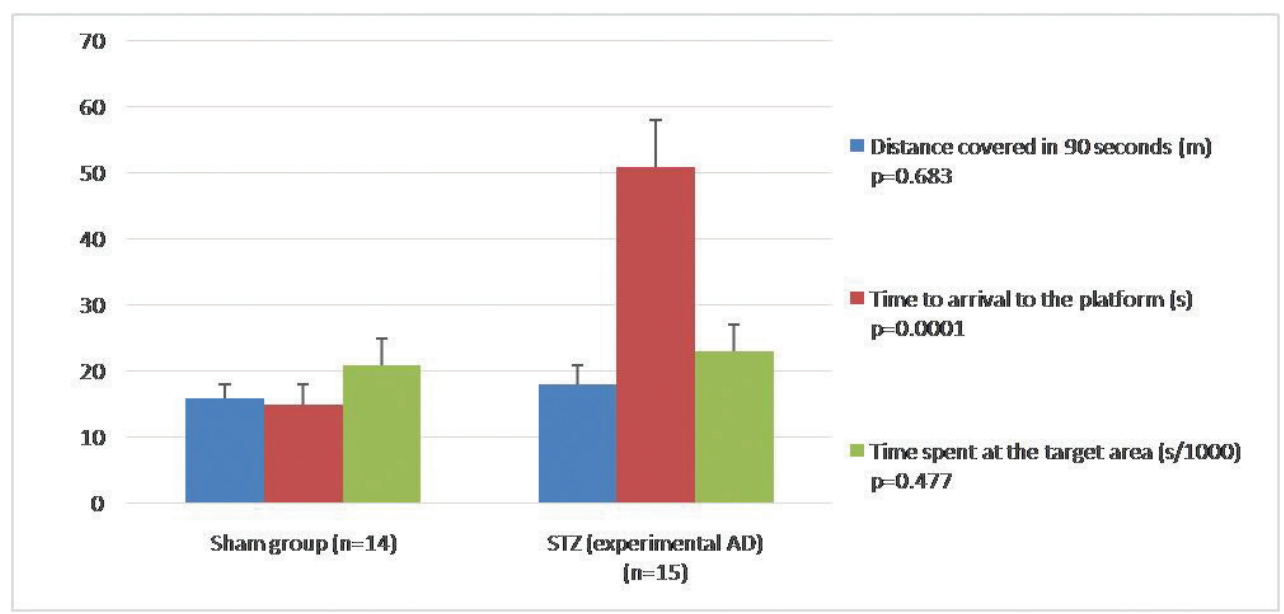

Figure 4. The results of the STZ and control groups on Water-Maze test before subdivision to treatment groups. The difference on time to arrival to the platform between the groups reached statistical significance but distance covered in 90 seconds and time spent in the target area did not (vertical axis shown in meters for distance covered in 90 second, seconds for time to arrival to the platform, and seconds/1000 for the time spent in the target area).

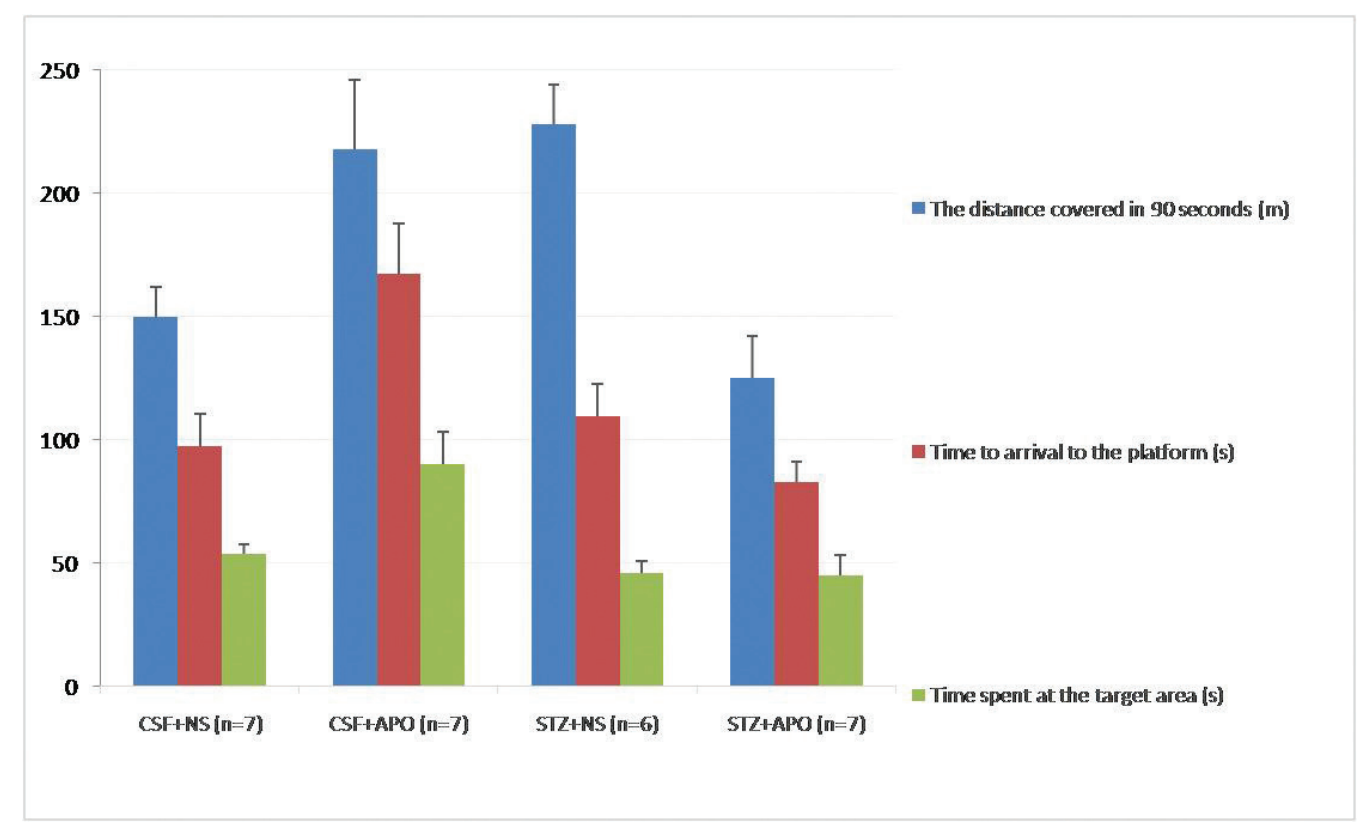

Figure 5. The results of Water-Maze tests. There were no statistically significant differences between all groups on distance covered in 90 seconds, time to arrival to the platform, and time spent in the target area (vertical axis shown in meters for distance covered in 90 second, seconds for time to arrival to the platform, and seconds/1000 for the time spent in the target area). 


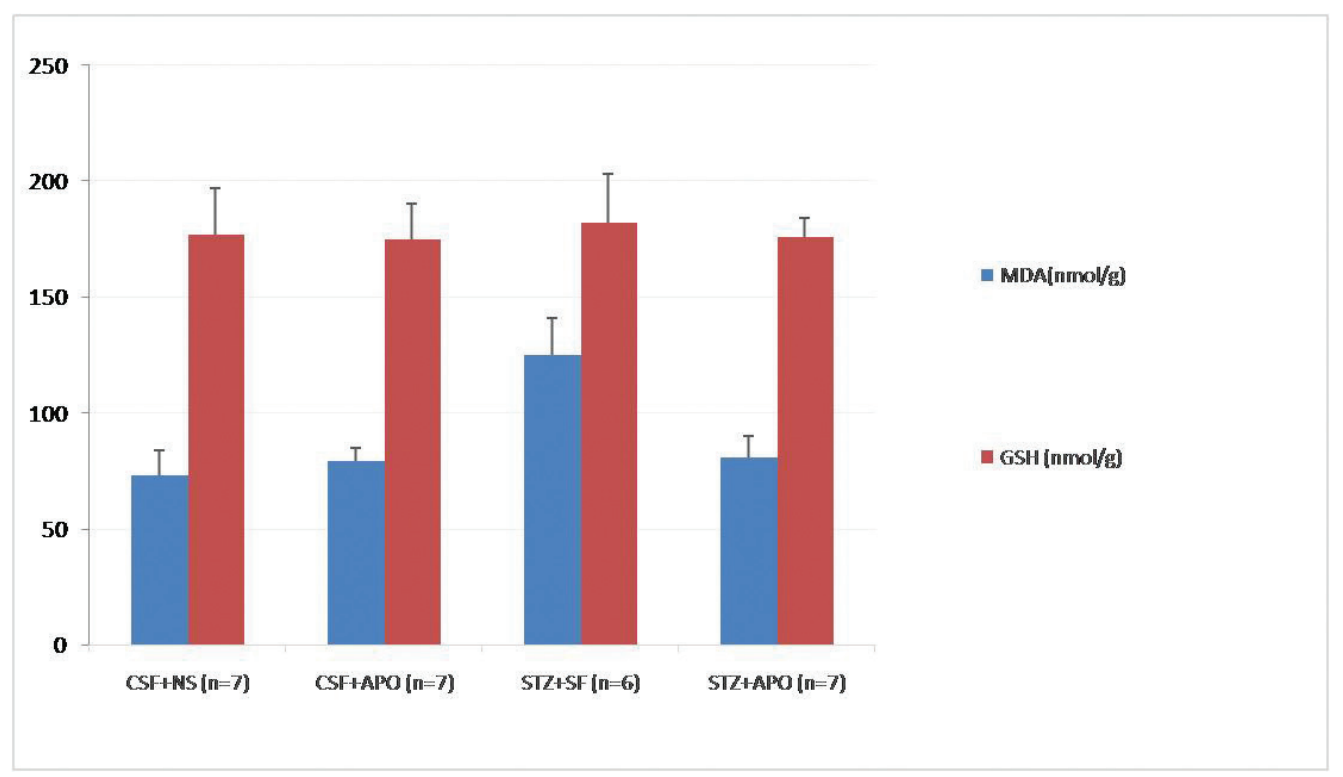

Figure 6. The results of MDA and GSH levels. The differences between the brain's GSH across the treatment groups were not statistically significant. MDA differences between $S T Z+A P O$ and $S T Z+N S$ groups reached statistical significance $(p=0.046)$ indicating the protective role of the APO (vertical axis shown in nmollg for MDA and (nmol/g) for GSH).

differences between $\mathrm{STZ}+\mathrm{NS}$ and $\mathrm{CSF}+\mathrm{NS}, \mathrm{CSF}+\mathrm{APO}$ and $S T Z+A P O$ and groups reached statistical significance $(\mathrm{p}<0.05)$ indicating the protective role of the APO (Figure 6).

\section{RESULTS OF AMYLOID DEPOSITION AND NEURAL DAMAGE}

A histologist blinded to treatment groups performed the histological examination. Light microscopy examina-
A

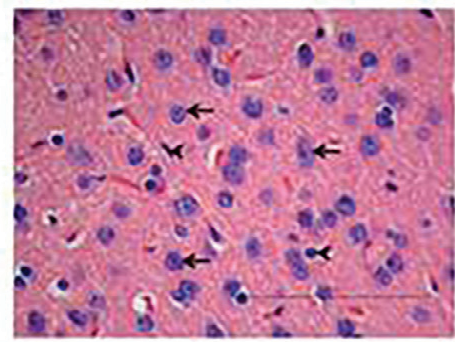

B

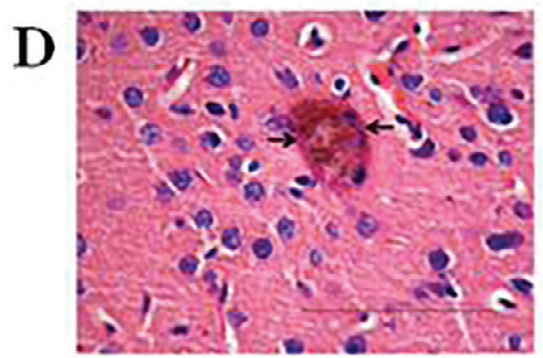

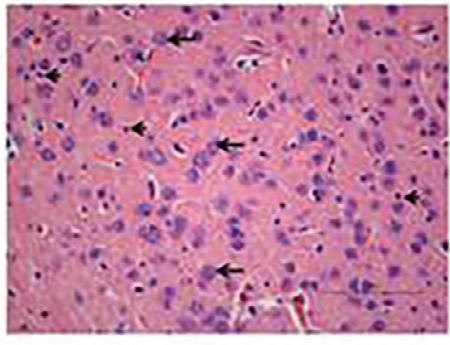

C

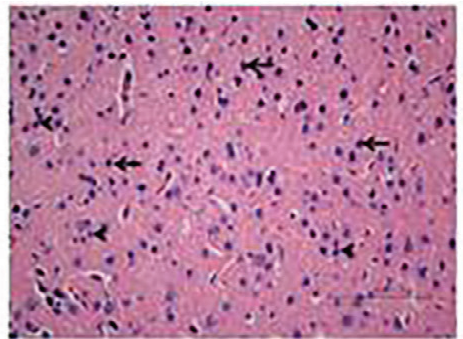

Figure 7. Light microscopy images in brain cortex.

A. CSF+NS. Brain cortex. Normal histological appearance. Neuronal nuclei (arrows), glial cells' nuclei (arrowheads). H\&E. Scale $100 \mu \mathrm{m}$. B. CSF+APO. Brain cortex. Histological appearance is very similar to normal. Neuronal nuclei (arrows), glial cell' nuclei (arrowheads). H\&E. Scale $100 \mu \mathrm{m}$. C. STZ+SF. Brain cortex. STZ induced neuronal damage. Pyknotic, heterochromatic, disorganized neuronal nuclei (arrows), increased glial cells (arrowheads). H\&E. Scale $100 \mu \mathrm{m}$. D. STZ+SF. STZ induced neuronal damage. Senile plaque formation in the brain cortex (arrow). H\&E. Scale $100 \mu \mathrm{m}$. E. STZ+APO. Brain cortex. STZ induced damage is partially reversed by APO. Pyknotic, heterochromatic, neuronal nuclei (arrows). H\&E. Scale $100 \mu \mathrm{m}$. 

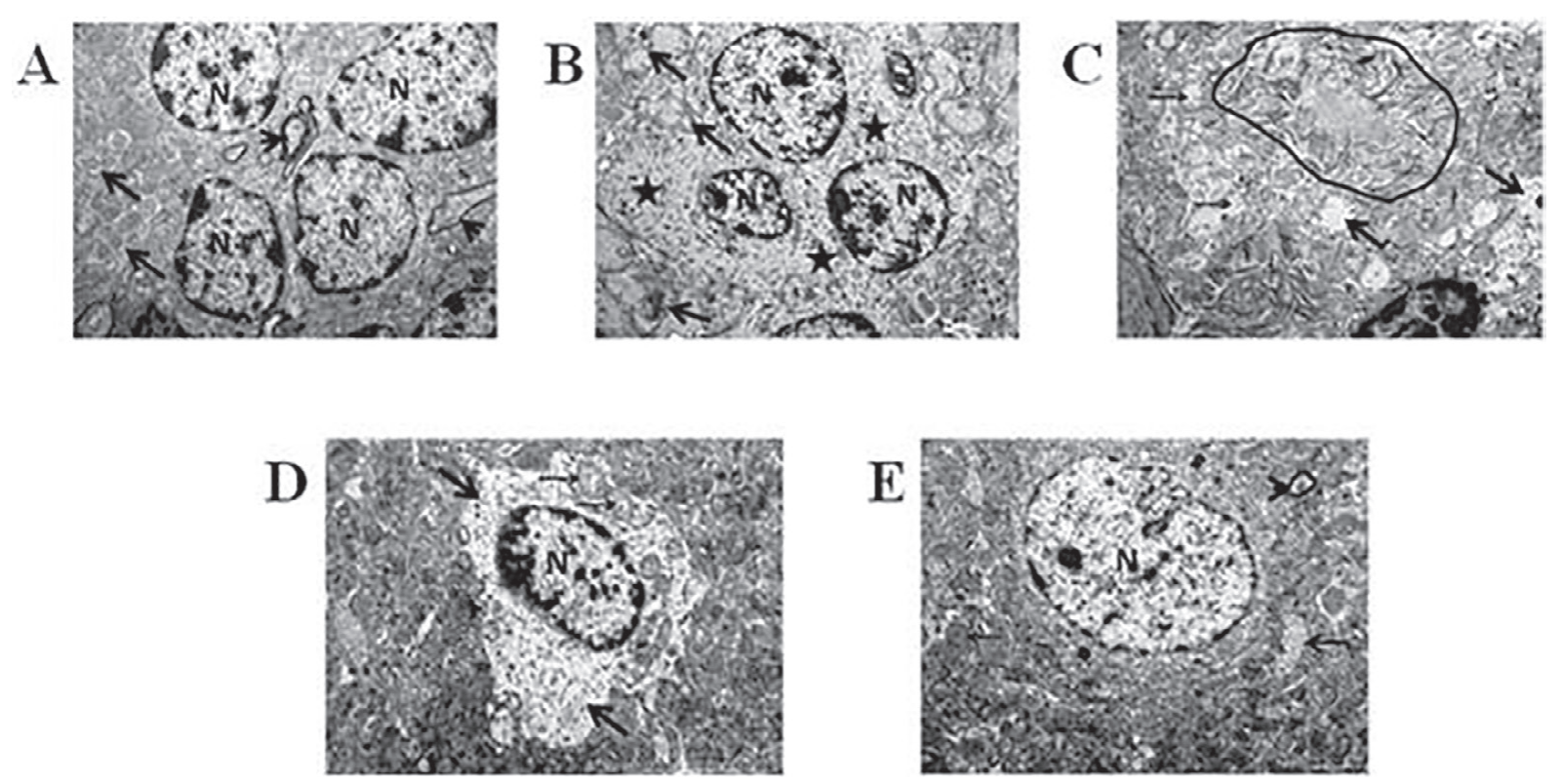

Figure 8. Images obtained from transmission electron microscope.

A. CSF+NS. Normal TEM appearance. Neuronal nucleus (N), dendritic neurofilaments and mitochondrion (arrow), myelinated axon (arrowhead). TEM. Scale $2 \mu \mathrm{m}$. B. STZ+NS. STZ induced organelle damage. Increased peripheral chromatin density in neuronal nucleus (N), intacytoplasmic edema and organelle degeneration (stars), neurofilament degeneration and loss in axons and dendrites (arrow). TEM. Scale 2 $\mu m$. C. STZ+NS. STZ induced organelle damage. Senile plaque (closed loop), loss of crista in mitochondria (thin arrow), neurofilament degeneration (thick arrow). TEM. Scale $2 \mu \mathrm{m}$. D. STZ+NS. STZ induced organelle damage. Condensation of peripheral chromatin in the neuronal nucleus $(N)$. Intra-cytoplasmic edema and organelle degeneration (thick arrows), mitochondrial damage and fusion in early stage of Charnoly body formation (thin arrows). TEM. Scale $2 \mu \mathrm{m}$. E. STZ+APO. STZ induced damage is partially reversed by APO. Although the damage is clearly visible it is not as severe as in $S T Z+N S$ group. Neuronal nucleus $(N)$, dendritic neurofilament (thick arrow), myelinated axon (arrowhead), mitochondrion (thin arrow). TEM. Scale $2 \mu \mathrm{m}$.

tion of $\mathrm{CSF}+\mathrm{NS}$ group was normal in histological appearance. Cortical layers were easily indefinable, neuronal nuclei were euchromatic and had clear contours (Figure $7 A)$. Neuronal/glia ratio was normal. Hippocampal granular and pyramidal neurons showed normal histological appearance. Under transmission electron microscopy neuronal cells had euchromatic and normally shaped nuclei (Figure 8A). Mitochondria were observed as oval crystals. Granular endoplasmic reticulum had normal ultrastructural appearance. There was an observable neurofilament web between glial cells and the neurons. The $\mathrm{H}-\mathrm{E}$ and TEM findings of the CSF-APO group were similar to that of CSF-NS (Figure 7B).

Light microscopy revealed numerous neurons with heterochromatic and irregularly shaped nuclei in STZ+NS group (Figure 7C and D). Neuron/glia ratio was decreased in the cortical areas. In addition, we noticed oval shaped necrotic neurons with sharp borders. Neurons in hippocampal area, especially pyramidal showed prominent heterochromasia, pyknosis, and degeneration. Neurofibriler tangles were observed in cortical and hippocampal areas. Some neurons had lucent perinuclear area that was interpreted as cytoplasmic edema. There were apoptotic neurons with piknotic nucleus and dense eosinophilic cytoplasm. Lipofuscin was present in some cortical cells. Additionally, senile plaques were present in cortical areas. TEM analysis in STZ+NS group showed the presence of auto-phagosomes, some filled with lipofuscin (Figure 8B and C). Axons and dendrites showed marked decrease in neurofilaments reaching total absence in some areas. Some neurons had concentration of peripheral chromatin density in addition to advanced intra-cytoplasmic edema and organelle degeneration. Mitochondria showed variable degrees of injury and subsequent degeneration in this group with subsequent fusion and formation of Charnoly bodies (Figure $8 \mathrm{D})$. There was disorganization, loss and degeneration of neurofilaments in neuronal soma and in between glial cells. Ultrastructural examination showed senile plaques with disorganized peripheral fibrils with homogenous center. STZ+APO group showed marked decrease in histological findings observed in STZ+NS group in both light microscopy and TEM (Figure 7E and 8E).

\section{DISCUSSION}

Despite intensive research the pathophysiology and treatment of AD remains elusive. Recently oxidative stress theory has gained interest in the scientific community. Oxidative stress has been thought one of the main reason that takes over a potential role in the pathogenesis of AD. 
Clinical and also preclinical researches show that neurodegenerative diseases such as $\mathrm{AD}$ are connected with higher levels of oxidative stress biomarkers and by lower levels of antioxidant defense biomarkers in the brain (24). Our study has been arised by this main topic.

Pradip explained connection between STZ and AD. $S T Z$ administration through route from intracebroventricular injection produces reduced cognition and increased cerebral aggregated $A \beta$ fragments, total tau protein, and $\mathrm{A} \beta$ deposits. These changes were accompanied with decreased glycogen synthase kinase (GSK-3) alpha/ beta ratio (phosphorylated/total) in the brain. Administration of STZ in a rodent's brain has been shown to produce neuroinflammation, oxidative stress and biochemical alterations, which is considered to be a valid experimental model of the early pathophysiological changes in neurodegenerative disease. STZ-induced spatial learning deficit in Morris water maze test and tau phosphorylation in rodent's brain produces sporadic $\mathrm{AD}(\mathrm{sAD})$ like pathology (25). For this reason, we selected to STZ-induced rat model to investigate protective effects of apocynin on AD.

In recent years, the application of protective agents to reduce the effects of endogenous oxidative stress and metabolic events related to external factors has been widespread $(2,3,16,17,18,21,26,27,28)$. It assumes that microglia reaction against amyloid plaques with subsequent release of reactive oxygen species is the major damaging factor $(20,29)$. Neuronal death in return leads to additional amyloid deposition and eventually vicious cycle repeats itself. Charnoly body has been shown to be a universal marker of neuronal damage $(30,31,32)$. Charnoly body is a pre-apoptotic and early biomarker of compromised mitochondrial bioenergetics and its observation in our animals supports this theory.

It has been suggested that free radical inhibition might be a therapeutic target. A lot of natural products which might achieve this mechanism are addressed for the treatment of AD. Besides neuroprotection, these products also reveal biological effects that target biochemical pathways underlying related signs of $\mathrm{AD}$ that include cognitive damages, mood and anxiety. $(33,34)$ Also researches indicated that NADPH oxidase is novel therapeutic targets for neurodegenerative disorders. NADPH oxidase seems to be specifically significant in the modulation of redoxsensitive signaling pathways and also has been implicated in neuronal dysfunction, degeneration, and neuroinflammation $(35,36)$. Gao et al. emphasized that if we inhibit neuronal NADPH oxidase activity we might treat patients who suffer from AD (36). For this reason, Apocynin is medicine which has recently gained popularity for $\mathrm{AD}$ but there is no enough study for this answer. That is why we made our study.

In the literature, we didn't find any research that $\mathrm{AD}$ was induced with ICV STZ and treated with IP APO. Lull et al have studied chronic oral APO in transgenic mice AD model (20). They gave same apocynin treatment as us which was $10 \mathrm{mg} / \mathrm{kg}$. Similar to our results the authors found decrease in amyloid plaque size and neuro-protective effect of APO. The results of the Water maze test however, showed no beneficial effect between the groups as in our study. In addition to the Water-Maze test, we performed rotarod and accelerod tests and found that the APO-treated mice performed better than control group on 30 and 40 but not at lower rpm's. It is probably due to the fact that the beneficial effect on motor function is subtle and therefore higher rotation speed is required to reveal it.

Dumont et al. found similar cognitive and histopathological results from the transgenic mouse model of $\mathrm{AD}$ who took also apocynin treatment and so emphasized that APO does not sufficient to ameliorate AD symptoms (37). Two animals from the STZ-NS group have died before the testing was completed. There were no deaths in other groups including (and most importantly) STZ-APO group. Although the mortality was not the end-result of our study this finding indicates that APO might also affect the mortality.

Some recent researches about $\mathrm{AD}$ were made from transgenic mouse models. These researches found that although there is a genetic susceptibility to $\mathrm{AD}$ in transgenic mouse, also there are an increased oxidative stress and neurodegenerative pathway which is similar to STZinduced models $(20,37,38)$. As our histopathological results, STZ-induced rat model is a reliable model, which might give us information about the motor and cognitive dysfunction of $\mathrm{AD}$ patients and their treatment.

APO's mechanism of action is a matter of controversy and has been linked to the inhibition of the reactive oxygen species formation. However, Lull et al found no difference in NADPH oxidase level between the APO-treated and control groups suggesting that the beneficial effect is independent of anti-oxidative action (20). Our results support anti-oxidative action of APO, since it restored the MDA level. Similarly Wilkinson et al attributed the protective role of ibuprofen on AD due to its inhibitory effect on NOX-2 (39). Further research is required to clarify the benefits of anti-oxidative treatment in AD.

In conclusion, APO showed beneficial effect on high challenge motor but not cognitive skills in STZ-induced model of AD. Histological and biochemical evaluations demonstrated APO's favorable effect.

\section{Conflict of interest}

None.

\section{REFERENCES}

1. HANLEY MR 1989 Peptide regulatory factors in the nervous system. Lancet 1(8651): 1373-1376. https://doi.org/10.1016/S0140-6736(89)92815-8

2. KIM GH, KIM JE, RHIE SJ, YOON S 2015 The Role of Oxidative Stress in Neurodegenerative Diseases. Experimental Neurobiology 24(4): 325-340. https://doi.org/10.5607/en.2015.24.4.325 
3. MONEIM AE 2015 Oxidant/Antioxidant imbalance and the risk of Alzheimer's disease. Current Alzheimer Research 12(4): 335 349. https://doi.org/10.2174/1567205012666150325182702

4. PETERSEN RC, THOMAS RG, GRUNDMAN M, BENNETT D, DOODY R, FERRIS S, GALASKO D, JIN S, KAYE J, LEVEY A, PFEIFFER E, SANO M, VAN DYCK CH, THAL LJ 2005 Alzheimer's Disease Cooperative Study: Vitamin E and donepezil for the treatment of mild cognitive impairment. England Journal of Medicine 352(23): 2379-2388. https://doi.org/10.1056/NEJMoa050151

5. PLUM L, SCHUBERT M, BRUNING JC 2005 The role of insulin receptor signaling in the brain. Trends Endocrinology Metabolism 16(2): 59-65. https://doi.org/10.1016/j.tem.2005.01.008

6. VIEIRA MNN, LIMA-FILHO RAS, DE FELICE FG 2017 Connecting Alzheimer's disease to diabetes: underlying mechanisms and potential therapeutic targets. Neuropharmacology S00283908(17): 30523-30533.

7. DE LA MONTE SM 2017 Insulin Resistance and Neurodegeneration: Progress Towards the Development of New Therapeutics for Alzheimer's Disease. Drugs 77(1): 47-65. https://doi.org/10.1007/s40265-016-0674-0

8. DE FELICE FG, LOURENCO MV, FERREIRA ST 2014 How does brain insulin resistance develop in Alzheimer's disease?. Alzheimer's \& Dementia 10.1: 26-32. https://doi.org/10.1016/j.jalz.2013.12.004

9. FERREIRA ST, CLARKE JR, BOMFIRM TR, DE FELICE FG 2014 Inflammation, defective insulin signaling, and neuronal dysfunction in Alzheimer's disease. Alzheimer's \& dementia 10(1): 76-83. https://doi.org/10.1016/j.jalz.2013.12.010

10. BEDSE G, DI DOMENICO F, SERVIDDIO G, CASSANO T 2015 Aberrant insulin signaling in Alzheimer's disease: current knowledge. Frontiers in neuroscience 16(9): 204. https://doi.org/10.3389/fnins.2015.00204

11. SZKUDELSKI T 2001 The mechanism of alloxan and streptozotocin action in B cells of the rat pancreas. Physiological Research 50(6): 537-546

12. SHARMA M, GUPTA YK 2001 Intracerebroventricular injection of streptozotocin in rats produces both oxidative stress in the brain and cognitive impairment. Life Science 68(9): 1021-1029. https://doi.org/10.1016/S0024-3205(00)01005-5

13. LANNERT H, HOYER S 1998 Intracerebroventricular administration of streptozotocin causes long-term diminutions in learning and memory abilities and in cerebral energy metabolism in adult rats. Behaviour Neuroscience 112(5): 1199-1208. https://doi.org/10.1037/0735-7044.112.5.1199

14. PETRONIO MS, ZERAIK ML, FONSECA LM, XIMENES VF 2013 Apocynin: chemical and biophysical properties of a NADPH oxidase inhibitor. Molecules 18(3): 2821-2839. https://doi.org/10.3390/molecules18032821

15. KLENIEWSKA P, PIECHOTA A, SKIBSKA B, GORACA A 2012 The NADPH oxidase family and its inhibitors. Archivum Immunologiae et Therapiae Experimentalis 60(4): 277-294. https://doi.org/10.1007/s00005-012-0176-z

16. CONNELL BJ, SALEH MC, KHAN BV, SALEH TM 2011 Apocynin may limit total cell death following cerebral ischemia and reperfusion by enhancing apoptosis. Food and Chemical Toxicology 49(12): 3063-3069. https://doi.org/10.1016/j.fct.2011.09.010

17. WANG Q, TOMPKINS KD, SIMONYI A, KORTHUIS RJ, SUN AY, SUN GY 2006 Apocynin protects against global cerebral ischemia-reperfusion-induced oxidative stress and injury in the gerbil hippocampus. Brain research 1090(1): 182-189. https://doi.org/10.1016/j.brainres.2006.03.060

18. AYDOGAN MS, PARLAKPINAR H, ERDOGAN MA, YUCEL A, UCAR M, SAGIR M, COLAK C 2013 Effects of dexme- detomidine and midazolam on motor coordination and analgesia: a comparative analysis. Current Therapy Research Clinical Experiment 75 : $22-26$.

https://doi.org/10.1016/j.curtheres.2013.05.003

19. NGUYEN TL, NAM YS, LEE SY, KIM HC, JANG CG 2010 Effects of capsazepine, a transient receptor potential vanilloid type 1 antagonist, on morphine-induced antinociception, tolerance, and dependence in mice. British Journal of Anaesthesia 105(5): 668 674. https://doi.org/10.1093/bja/aeq212

20. LULL ME, LEVESQUE S, SURACE MJ, BLOCK ML 2011 Chronic apocynin treatment attenuates beta amyloid plaque size and microglial number in hAPP(751)(SL) mice. PloS one 6(5): e20153. https://doi.org/10.1371/journal.pone.0020153

21. ISIK AT, CELIK T, ULUSOY G, ONGORU O, ELIBOL B, DORUK H, BOZOGLU E, KAYIR H, MAS MR, AKMAN S 2009 Curcumin ameliorates impaired insulin/IGF signalling and memory deficit in a streptozotocin-treated rat model. Age 31(1): 39-49. https://doi.org/10.1007/s11357-008-9078-8

22. PAXINOS G, ASHWELL KW, TORK I 2013 Atlas of the developing rat nervous system. Academic Press.

23. YILDIZ SE, DEPREM T, SARI EK, BINGOL SA, TASCI SK ASLAN S, NUR G, SOZMEN M 2015 Immunohistochemical distrubition of leptin in kidney tissues of melatonin treated diabetic rats. Biotechnic\&Histochemistry 90(4): 270-277. https://doi.org/10.3109/10520295.2014.983548

24. NIEDZIELSKA E, SMAGA I, GAWLIK M, MONICZEWSKI A, STANKOKICZ P, PERA J, FILIP M 2016 Oxidative stress in neurodegenerative diseases. Molecular Neurobiology 53(6), 4094 4125. https://doi.org/10.1007/s12035-015-9337-5

25. PRADIP KK 2015 Streptozotocin induced Alzheimer's disease like changes and the underlying neural degeneration and regeneration mechanism. Neural Regeneration Research 10(7): 1050. https:// doi.org/10.4103/1673-5374.160076

26. NUR G, DEVECI HA, ERSAN Y, MERHAN O, NAZLI M, NUR O 2016 Protective Role of Caffeic Acid Phenethyl Ester against Tetramethrine-Induced Toxicity in Mice. Medical Science 5(4): 972-8. https://doi.org/10.5455/medscience.2016.05.8489

27. DEVECI HA, NUR G, ALPAY M, OZMERDIVENLI R 2017 Levels of paraoxonase, high-density lipoprotein and total sialic acid in patients with polycystic over syndrome Journal of Cellular Neuroscience \& Oxidative Stress 9(2): 630-636.

28. DEVECI HA, KARAPEHLIVAN M, KAYA I, KUKURT A, ALPAY M 2015 Akut klorprifos-etil zehirlenmesine karşı kafeik asit fenetil ester' in koruyucu etkisi(Defense effect of caffeic acid phenethyl ester against acute chlorpyrifos-ethyl poisoning). Ankara Üniversitesi Veterinerlik Fakültesi Dergisi 62: 255-260. https://doi.org/10.1501/Vetfak_0000002689

29. YALABIK MS, SEHIRLI O, UTKAN T, ARICIOGLU F 2013 Effects of agmatine in streptozotocine induced experimental alzheimer model. Marmara Üniversitesi SBE Dergisi 3(3): 145.

30. JAGTAP A, GAWANDE S, SHARMA S 2015 Biomarkers in vascular dementia: A recent update. Biomarkers Genomic Medicine 7(2): 43-56. https://doi.org/10.1016/j.bgm.2014.11.001

31. SHARMA S, EBADI M 2014 The Charnoly body as a universal biomarker of cell injury. Biomarkers Genomic Medicine 6(3): 89-98. https://doi.org/10.1016/j.bgm.2014.03.004

32. SHARMA S, MOON CS, KHOGALI A, HAIDOUS A, CHABENNE A, OJO C, JELEBINKOW M, KURDI Y, EBADI M 2013 Biomarkers in Parkinson's disease (recent update). Neurochemistry international 63(3): 201-229.

https://doi.org/10.1016/j.neuint.2013.06.005

33. DIVINO da ROCHA M, PEREIRA DIAS VIEGAS F, CRISTINA CAMPOS H, CAROLINA NICASTRO P, CALVE FOSSALUZZA P, ALBERTO MANSOUR FRAGA C, BARREIRO E, VIEGAS C 2011 The role of natural products in the discovery 
of new drug candidates for the treatment of neurodegenerative disorders II: Alzheimer's disease. CNS Neurol Disord Drug Targets 10(2): 251-270. https://doi.org/10.2174/187152711794480429

34. KUMAR A, SINGH A 2015 A review on mitochondrial restorative mechanism of antioxidants in Alzheimer's disease and other neurological conditions. Frontiers in pharmacology 6 . https://doi.org/10.3389/fphar.2015.00206

35. MALDONADA PD, MOLINA JIJóN E, VILLEDA HERNáANDEZ J, GALVáN ARZATE S, SANTAMARIA A, PEDRAZA CHAVERRI J 2010 NADPH oxidase contributes to neurotoxicity in an excitotoxic/prooxidant model of Huntington's disease in rats: protective role of apocynin. Journal of neuroscience research 88(3): $620-629$.

36. GAO HM, ZHOU H, HONG JS 2012 NADPH oxidases: novel therapeutic targets for neurodegenerative diseases. Trends Pharmacollogical Science 33(6): 295-303.

https://doi.org/10.1016/j.tips.2012.03.008
37. DUMONT M, STACK C, ELIPENHALI C, CALINGASAN NY, WILLE E, BEAL MF 2011 Apocynin administration does not improve behavioral and neuropathological deficits in a transgenic mouse model of Alzheimer's disease. Neuroscience letter 492(3): 150-154. https://doi.org/10.1016/j.neulet.2011.01.077

38. HASHIMOTO Y, ITO Y, ARAKAWA E, KITA Y, TERASHITA K, NJIKURA T, NISHIMOTO I 2002 Neurotoxic mechanisms triggered by Alzheimer's disease linked mutant M146L presenilin 1: involvement of $\mathrm{NO}$ synthase via a novel pertussis toxin target. Journal of Neurochemistry 80(3): 426-437. https://doi.org/10.1046/j.0022-3042.2001.00722.x

39. WILKINSON BL, CRAMER PE, VARVEL NH, REEDGEAGHAN E, JIANG Q, SZABO A, HERRUP A, LAMB BT, LANDRETH GE 2012 Ibuprofen attenuates oxidative damage through NOX2 inhibition in Alzheimer's disease. Neurobiology of Aging 33(1): 197.e121-197.e132.

https://doi.org/10.1016/j.neurobiolaging.2010.06.014 